\title{
Case Report: Segmental Testicular Infarction
}

\author{
Muhammad Imran Aumeerally* \\ Department of Surgery, Mackay Base Hospital, West Mackay, Queensland, Australia
}

*Corresponding author: Muhammad Imran Aumeerally, Department of Surgery, 475 Bridge Road, Mackay Base Hospital, West Mackay, Queensland, 4740, Australia, Tel: +61408186991; E-mail: imran.aumeerally@health.qld.gov.au

Received: 28 Sep, 2019 | Accepted: 14 Oct, 2020 | Published: 20 Oct, 2020

Citation: Aumeerally MI (2020) Case Report: Segmental Testicular Infarction. J Surg Open Access 6(6): dx.doi.org/10.16966/2470-0991.227

Copyright: (c) 2020 Aumeerally MI. This is an open-access article distributed under the terms of the Creative Commons Attribution License, which permits unrestricted use, distribution, and reproduction in any medium, provided the original author and source are credited.

\begin{abstract}
Segmental testicular infarction is a rare condition that presents with acute testicular pain. The diagnosis can be clinically challenging and include important differential diagnoses such as testicular torsion, testicular tumour and infection. This case report describes a 27-year-old male presenting to a regional Australian hospital with acute left testicular pain. The diagnosis was made intra-operatively during surgical exploration and on histopathologic assessment after the patient underwent a partial orchidectomy. While segmental testicular infarction is a rare condition, it should be considered in the differential diagnosis for acute testicular pain as awareness may help to avoid unnecessary radical surgery.
\end{abstract}

Keywords: Infarct; Segmental; Testis

\section{Introduction}

Segmental testicular infarction is a rare condition with fewer than 100 case reports in the English literature [1]. It is a rare cause of acute testicular pain which may mimic more common pathologies such as torsion and infection. Due to the low volume of cases reported, there is no consensus on conservative versus operative management of this condition. The aetiology is also unclear although trauma and torsion are potential causes.

\section{Case Report}

A 27-year-old male patient presented to a rural Australian hospital with a 12-hour history of acute a traumatic left hemi-scrotal pain. He had no previous significant past medical or surgical history. On examination, he had exquisite tenderness at the inferior pole of the left testicle without any other abnormality identified. No laboratory investigations were ordered. Ultrasound was performed and demonstrated normal sonographic appearance of the testicles bilaterally.

Given the ongoing pain, the decision was made to proceed to a scrotal exploration under general anaesthesia. Intra-operative findings (Figure 1) revealed a segmental patch of ischaemia of the left testicle and a partial orchidectomy with suture orchidopexy was performed. The patient recovered without complication and was discharged at 1-day post-operation.

Histopathology confirmed partial testicular infarction and there was no evidence of malignancy or neoplasm. The patient was reviewed in clinic 1-month post-operation. He had no post-operative issues and was discharged back to the community.

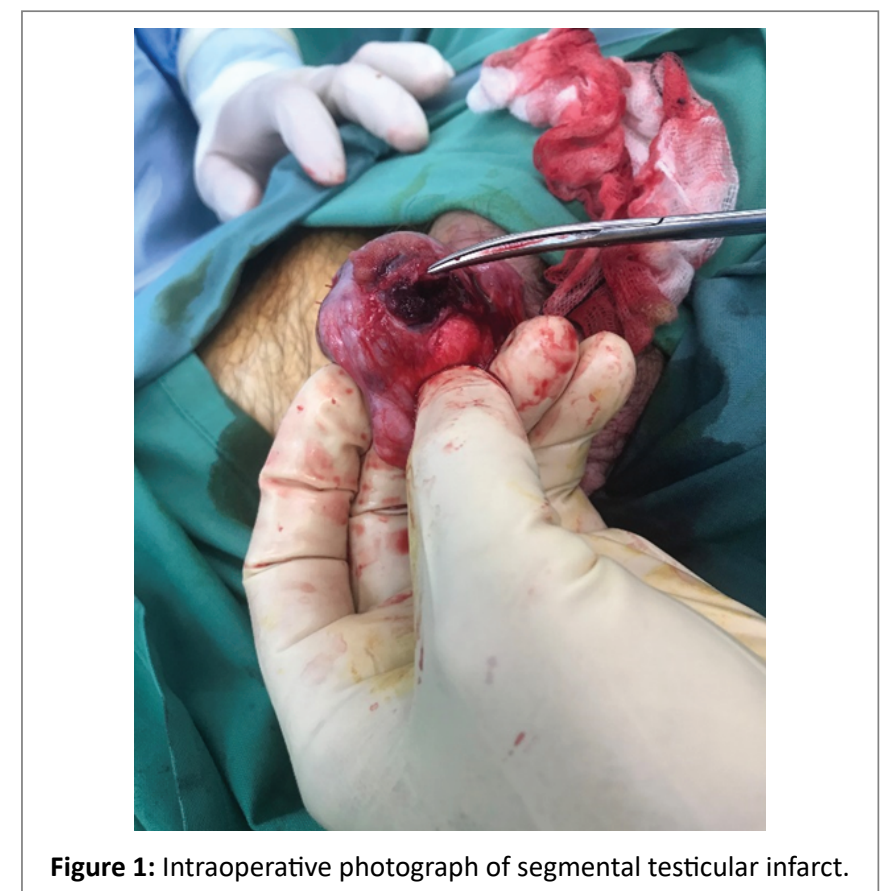

\section{Discussion and Conclusion}

Segmental testicular infarction is a rare condition of undetermined aetiology. Cases are often idiopathic although there are case reports suggesting a history of trauma, the presence of infection or an underlying vasculitis may be potential causes $[2,3]$. 
The most common symptom in segmental testicular infarction is acute testicular pain. Laboratory investigations are often normal. Ultrasound sonography may occasionally demonstrate a hypoechogenic lesion within the testicle which often includes neoplasm as a differential diagnosis. Ultimately, the diagnosis is made intra-operatively.

In younger patients, a partial orchidectomy may be performed if feasible. Otherwise, unilateral orchidectomy may be safely performed in older patients or those who have completed their families and are not concerned with cosmesis or function.

Early surgical intervention will expedite diagnosis leading to earlier recovery and with a low risk of complication.

\section{References}

1. Ernst S, Saar M, Brenneis H, Kubale R, Ueberdiek S, et al. (2018) Segmental testicular infarction: Case series and literature review of a rare diagnosis in men with acute testicular pain. Urol Int 101: 114116.

2. Gkolezakis V, Petrolekas A, Koutsouri A, Ouranos V, Arapantoni P, et al. (2018) Segmental testicular infarction. Urologe A 57: 947-950.

3. Karabulut I, Yılmaz AH, Koç M, Demirdöğen SO (2017) A Rare Case: Segmental Testicular Infarction. İstanbul Med J 18: 236-238. 